\title{
Trends in Radiation Practices for Female Ocular Oncologists in North America: A Collaborative Study of the International Society of Ocular Oncology
}

\author{
Sona N. Shah ${ }^{\mathrm{a}, \mathrm{b}}$ Kaitlin Kogachi ${ }^{\mathrm{a}, \mathrm{b}}$ Zelia M. Correa ${ }^{\mathrm{c}}$ Amy C. Schefler ${ }^{\mathrm{d}}$ \\ Mary E. Aronow ${ }^{e}$ Sonia A. Callejo ${ }^{f}$ Colleen M. Cebulla ${ }^{g}$ \\ Shelley Day-Ghafoori ${ }^{\text {h }}$ Jasmine H. Francis ${ }^{i}$ Sara Lally ${ }^{j}$ \\ Tara A. McCannel ${ }^{k}$ Katherine E. Paton' Isabella T. Phan ${ }^{\mathrm{m}}$ \\ Renelle Pointdujour-Lim ${ }^{\mathrm{n}}$ Aparna Ramasubramanian ${ }^{\circ}$ Pamela Rath $^{p}$ \\ Carol L. Shields ${ }^{j}$ Alison H. Skalet ${ }^{q}, r \quad$ Jill R. Wells ${ }^{s}$ Richard L. Jennelle ${ }^{t}$ \\ Jesse L. Berry ${ }^{\mathrm{a}, \mathrm{b}}$ for the Women in Ocular Oncology Consortium
}

a USC Roski Eye Institute, University of Southern California, Los Angeles, CA, USA; ${ }^{b}$ Children's Hospital Los Angeles, Los Angeles, CA, USA; ' University of Cincinnati, Cincinnati, OH, USA; ${ }^{d}$ Retina Consultants of Houston, Houston, TX, USA; ${ }^{~}$ Massachusetts Eye and Ear, Retina Service, Harvard Medical School, Boston, MA, USA; ${ }^{f}$ University of Montreal, Montreal, QC, Canada; ${ }^{9}$ Ohio State University, Columbus, OH, USA; ${ }^{\text {h}}$ Austin Retina Associates, Austin, TX, USA; 'Memorial Sloan Kettering Cancer Center, New York City, NY, USA; 'Ocular Oncology Service, Wills Eye Hospital,

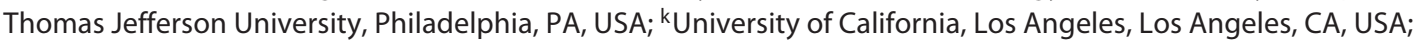
'University of British Columbia, Vancouver, BC, Canada; ${ }^{\mathrm{m}}$ Kaiser Permanente, Northern California, San Francisco,

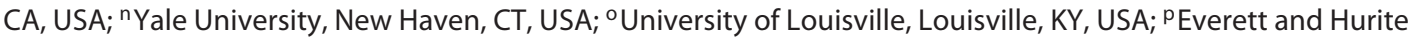
Ophthalmic Association, Pittsburgh, PA, USA; ${ }^{9}$ Casey Eye Institute, Oregon Health \& Science University, Portland, OR, USA; ' Department of Radiation Oncology, Oregon Health \& Science University, Portland, OR, USA; ${ }^{5}$ Emory Eye Center, Emory University, Atlanta, GA, USA; ${ }^{\mathrm{t}}$ Department of Radiation Oncology, University of Southern California, Los Angeles, CA, USA

\section{Keywords}

Melanoma - Choroidal neoplasms - Plaque brachytherapy · Radiation exposure $\cdot$ Female surgeons $\cdot$ Women surgeons

\footnotetext{
Abstract

Background: The aim of this study was to determine the known radiation exposure, attitudes, and consequent risk modifications among female ocular oncologists in North America who routinely administer radioactive plaque
}

brachytherapy treatment and are members of the International Society of Ocular Oncology. Methods: Nineteen female ocular oncologists completed an anonymous 17-question radiation exposure survey. Results: Eleven of the participants chose to routinely wear lead protection during surgery; 8 did not. Fifteen of 19 participants reported using an unloaded "nonactive" template to prepare for plaque implantation. During pregnancy, 11 of 13 participants continued to perform plaque brachytherapy. Eight of these 11 undertook measures to decrease radiation exposure self-re-

\section{KARGER}

(c) 2018 S. Karger AG, Basel

E-Mail karger@karger.com

www.karger.com/oop
Jesse L. Berry, MD

USC Roski Eye Institute

1450 San Pablo Street

Los Angeles, CA 90033 (USA)

E-Mail jesse.berrymd@ gmail.com 
ported as lead wear and other. The average reported anxiety regarding fertility was 2.1 (SD, 2.2) on a scale from 1 to 10. Conclusion: This study corroborates prior literature that surgeons' exposure to radiation during plaque brachytherapy is minimal. Nonetheless, there remains some anxiety regarding exposure risk to women, due to potential effects on fertility and fetal health. We found variability in exposure monitoring, required training, and precautions during pregnancy amongst this group of surgeons. Improved education and clearer pregnancy guidelines may equip female ocular oncologists with optimal knowledge regarding risk of radiation exposure.

(c) 2018 S. Karger AG, Basel

\section{Introduction}

In the field of ocular oncology, radiotherapy is essential to the treatment of ocular and orbital tumors, including various forms, such as external bean radiotherapy, proton beam therapy, and plaque brachytherapy [1-6]. Plaque brachytherapy involves surgical placement of a radioactive plaque onto the sclera adjacent to the tumor base in order to deliver a concentrated dose of radiation to the tumor while sparing surrounding ocular structures as much as possible [4]. Iodine, ruthenium, and palladium radioisotopes are commonly utilized for treating uveal melanoma and may also be used for treating invasive squamous cell carcinoma, metastatic choroidal lesions, retinoblastoma, and some benign retinal and choroidal tumors $[3,4]$. The total dose to which the surgeon is exposed depends upon the plaque type, overall radiation dose loaded in the plaque, surgical time with the active "hot" plaque, and proximity to the unshielded side of the plaque $[5,7]$. The key components in radiation protection are operative duration, distance, and shielding [3].

The National Council on Radiation Protection and Measurements (NCRP) advises the government on recommended maximum safe exposure limits, which are delineated in the Code of Federal Regulations [8]. These limits are expressed in the SI unit of Sievert (Sv), which is functionally equivalent to Gray (Gy), for purposes associated with eye plaques $[8,9]$. Table 1 summarizes the currently accepted annual exposure values which are $10 \times$ greater for the hands than for the body dose.

There are no gender differences for exposure guidelines with the same total body dose for men and women; however, exposure rates are more stringent for pregnant women. While exposure of the embryo-fetus to doses $\leq 100 \mathrm{mSv}$ are unlikely to cause any harm to the child
Table 1. Currently accepted annual radiation exposure limits $[8,9]$

Equivalent doses, $\mathrm{mSv} v^{\mathrm{a}}$

$\begin{array}{lc}\text { Total body dose } & 50 \\ \text { Intraocular lens dose } & 150 \\ \text { Hand dose } & 500 \\ \text { Total dose limit embryo-fetus } & 5 \\ \text { Monthly dose limit embryo-fetus } & 0.5 \\ \text { Negligible individual risk level } & 0.01\end{array}$

a $1 \mathrm{mSv}=0.1 \mathrm{rem}=0.001 \mathrm{~Gy} ; 1 \mathrm{~Sv}=1 \mathrm{~Gy}$.

[8-11], the dose limit to the fetus is recommended at $<5$ $\mathrm{mSv}$.

The aim of this study was to examine the risk modification and attitudes towards radiation exposure specifically among North American female ocular oncology surgeons who routinely administer radioactive plaque brachytherapy treatment. By determining the concerns and precautions taken by female surgeons who are routinely exposed to radiation, we can better equip these surgeons with the information and guidelines needed to feel safe in their line of work and the mechanisms to protect their patients and themselves from unnecessary radiation exposure.

\section{Methods}

This was an investigator-initiated survey study. Qualtrics software (Provo, UT, USA) was used to construct an online survey consisting of 17 questions addressing radiation exposure concerns and consequent precautions. The survey was distributed via e-mail to North American female ocular oncologists registered as members of the International Society of Ocular Oncology (ISOO) who regularly administer episcleral radioactive plaque brachytherapy treatment in their clinical practice (e.g., the primary surgeon for both plaque placement and plaque removal). The e-mail link was e-mailed to participants who could access the survey anonymously. Participants first confirmed their roles as female ocular surgeons actively using plaque brachytherapy in clinical practice; if this criterion was fulfilled, the participants were allowed to continue the survey. Participants could choose to remain anonymous and could skip any number of questions while still completing the survey. Authorship was optional. This study was approved by the University of Southern California Institutional Review Board.

Demographic information was collected, including age group, practice type, specialty, and location of practice in North America. Participants were also asked about the required radiation safety training at their institutions and the frequency of refresher training courses. Other information queried included personal measured radiation exposure, such as the use of a ring or button to track an- 
Table 2. Demographics and responses of survey participants

\begin{tabular}{|c|c|}
\hline All participants & 19 \\
\hline \multicolumn{2}{|l|}{ Age } \\
\hline$<40$ years & 8 \\
\hline $40-49$ years & 9 \\
\hline $50-59$ years & 2 \\
\hline \multicolumn{2}{|l|}{ Practice type } \\
\hline Academic institution & 14 \\
\hline Private practice & 5 \\
\hline \multicolumn{2}{|l|}{ Specialty } \\
\hline Oncology & 10 \\
\hline Retina/oncology & 7 \\
\hline Pediatrics/oncology & 1 \\
\hline Plastics/oncology & 1 \\
\hline \multicolumn{2}{|l|}{ Location } \\
\hline Northeast & 6 \\
\hline West & 4 \\
\hline Midwest & 3 \\
\hline South & 4 \\
\hline Canada & 2 \\
\hline \multicolumn{2}{|l|}{ Required radiation safety training } \\
\hline Yes & 11 \\
\hline \multicolumn{2}{|l|}{ Required refresher radiation safety course } \\
\hline Yes & 2 \\
\hline \multicolumn{2}{|l|}{ Ring or button to track radiation exposure } \\
\hline Yes & 14 \\
\hline \multicolumn{2}{|l|}{ Yearly radiation dose } \\
\hline Unknown & 12 \\
\hline Minimal & 4 \\
\hline Other & 3 \\
\hline \multicolumn{2}{|c|}{$\begin{array}{l}\text { Radiation protection during eye plaque placement } \\
\text { (irrespective of pregnancy) }\end{array}$} \\
\hline None & 8 \\
\hline Lead apron & 3 \\
\hline Lead skirt & 1 \\
\hline Neck guard & 1 \\
\hline Lead skirt, lead apron, neck guard & 6 \\
\hline
\end{tabular}

nual exposure, yearly radiation dose (if known), utilization of radiation protection while placing and removing plaques, precautions taken during administration of plaque brachytherapy to lower radiation exposure, type of plaque used, and type of surgical planning. For questions regarding the use of radiation protection and precautions during administration of plaque brachytherapy, participants could select multiple options.

Questions on radiation protection during pregnancy (if applicable) were also included. Participants were asked about any changes in practice before pregnancy and whether they continued to administer radioactive plaque brachytherapy treatment during pregnancy. If participants continued to perform such procedures during pregnancy, they were asked about protective measures undertaken to minimize radiation exposure. If these questions were not applicable, participants could select "NA." On a scale from 0 to 10 , with 10 representing the maximum level, participants rated their level of anxiety regarding fertility as related to their radiation exposure.
Precautions to lower radiation dose (irrespective of pregnancy)

Changing the sequence of surgery to decrease exposure time

Increasing the surgical distance

Decreasing the surgical time

Avoid having patients come to the office or rounding on patients while plaques are in place $\quad 6$

Type of radiation eye plaque

Eye physics

COMS

Other

Timing of plaque placement planning $\begin{array}{ll}\text { Preoperative plaque placement planning } & 7\end{array}$ Intra-operative plaque placement planning $\quad 5$ Both equally Other

Practice patterns with pregnancy

Participants with a history of pregnancy

Surgeons choosing to continue placing plaques during pregnancy

Yes

No

Increased protective measures during pregnancy $\quad 11$ Yes No

Protective measures during pregnancy Lead Apron/Skirt Other

Using the online Qualtrics software, data was collected and anonymously exported as an Excel spreadsheet (Microsoft, Redmond, WA, USA). The data was analyzed using Microsoft Excel, and percentages were calculated to represent the frequency of responses.

\section{Results}

A total of 25 female ocular oncologists in North America were identified by membership of the ISOO and e-mailed. Twenty-three of 25 women responded ( $92 \%$ response rate), and 19 of the 25 women regularly perform plaque brachytherapy and thus completed the survey ( $76 \%$ completion rate); 2 did not qualify to com- 
plete the survey and 2 did not answer all questions and so were excluded from the study. Thirteen of the 19 women who were included in the study had previously been pregnant. Demographics and responses are displayed in Table 2. Eight participants were $<40$ years old, 9 participants were in the range of 40-49 years old, and 2 participants were in the range of 50-59 years old. Fourteen participants practiced in academic institutions, and 5 were in private practice. Ten participants were in a subspecialty ocular oncology practice, 7 practiced both retina and oncology, 1 practiced pediatrics and oncology, and 1 practiced plastics and oncology. Six participants were from the Northeast, 4 were from the West, 3 were from the Midwest, 4 were from the South, and 2 were from Canada.

Eleven participants were required to take a radiation safety course through their institution. Two participants had taken mandatory refresher courses for radiation safety training, and 1 reported a requirement for annual recertification. Fourteen participants reported wearing a radiation dosimeter (ring and/or body sensor) to monitor their radiation exposure; 3 participants reported doses ranging from $<1$ to $20 \mathrm{mSv}$, while the other 4 had exposure reports of 0 or "minimal" per their institutional reports. Eleven participants chose to wear either a lead skirt, lead apron, neck guard, or all 3 ; and 8 participants did not use lead protection during surgery. Fifteen of 19 reported using a nonactive plaque to prepare for administration of plaque brachytherapy treatment, and then using the active plaque only after all preparations were complete. It must be noted that this decision to utilize a nonactive plaque may have been driven by the incentive to reduce radiation exposure; however, this was not specifically queried.

When asked about precautions to lower radiation dose irrespective of pregnancy, 7 participants selected multiple answers. Thirteen participants reported changing the sequence of surgery with examples including biopsy before administration of radioactive plaque brachytherapy treatment or confirmation of plaque location with an unloaded "nonactive" plaque before handling the radioactive plaque. Four participants increased their surgical distance from the eye, 5 participants decreased the surgical time whenever possible, and 6 participants avoided close proximity to patients with radioactive plaques postoperatively. Four participants did not respond to this question. Seven participants reported preoperative planning as their primary method for planning of plaque brachytherapy treatment, 5 reported intra-operative planning, and 4 reported using both preoperative and intra-operative

Trends in Radiation Practices for Female

Ocular Oncologists in North America planning equally. One participant reported only intraoperative planning without using a nonactive plaque but with ultrasound confirmation of hot plaque administration.

Thirteen women answered questions related to childbearing; 6 participants stated that they had not been pregnant. Eleven of the 13 who responded continued to perform plaque brachytherapy treatments while pregnant and 2 stopped. Eight of these 11 participants changed their plaque brachytherapy treatment administration practices during pregnancy with 7 participants wearing a lead apron or skirt and the other participant changing her practice of localizing and placing the scleral sutures but not tying down the active plaque (a colleague was available for this part of the operation). One of the 3 participants who did not change her plaque brachytherapy administration practices during pregnancy reported habitually wearing protective gear, including a lead skirt, apron, and neck guard. Participants also rated their anxiety regarding fertility on a scale from 0 to 10 with 10 being the highest anxiety level; the average reported anxiety was $2.1(\mathrm{SD}, 2.2)$.

\section{Discussion}

Overall, administration of radioactive plaque brachytherapy treatment is considered a safe procedure for surgeons to perform, with radiation exposure that does not exceed accepted standards. However, radiation exposure for pregnant women must conform to an even higher level of precaution [10,12-15]. There are few female ocular oncologists who perform plaque brachytherapy regularly in North America; therefore, we aimed to evaluate their practice trends, overall level of exposure, and consequent concern. An average individual receives a radiation dose of $0.01 \mathrm{mSv}$ daily from background environmental exposure, $0.02 \mathrm{mSv}$ from a chest X-ray, and 0.04 $\mathrm{mSv}$ from cosmic exposure on an airplane flight from New York to Los Angeles [9]. The maximum allowable radiation exposure is $50 \mathrm{mSv} /$ year to the whole body and $500 \mathrm{mSv}$ limited to the hand, as a much higher dose is tolerated by the extremities [8]. In comparison, of the 8 participants who monitored their yearly radiation exposure, 3 reported doses ranging from $<1$ to $20 \mathrm{mSv}$, and the other 4 reported 0 or "minimal" per their institution. With 1 exception, none of the reported values would have exceeded the allowed dose of $5 \mathrm{mSv}$ to the fetus during gestation. No health effects following exposure in utero have been noted for doses $<50 \mathrm{mSv}$, which is 10 
times higher than the allowable dose of $5 \mathrm{mSv}$ during gestation. Only 1 reported dose from this cohort of female ocular oncologists was higher than the gestational limit, and all were less than the body dose of $50 \mathrm{mSv}$ [16]. While there is theoretical concern about fetal exposure in surgeons, pregnant women who have required brachytherapy have been reported, and there have been no radiation-related issues to the fetus $[16,17]$. Nonetheless, this is a very small cohort of women with reported fetal exposure, and the health effects of very low-dose radiation remain an area of hot controversy. Our best guidance comes from the BEIR VII report [18], and currently the standard model is the linear no threshold (LNT) model. However, the biologic effects of radiation doses on the order of background radiation $(\sim 13 \mathrm{mSv} /$ year $)$ have not been shown to result in any biologically meaningful issues. Furthermore, occupational exposure limits are designed so that the risks involved from occupational exposure are chosen to be "as safe as the safest" limits in other areas of risk [18].

Regardless, the negative outcomes due to radiation overexposure have inspired research on the safety of health-care professionals in a variety of fields. Laube et al. [19] examined the radiation from iodine-125 and ruthenium-106 plaques on the fingers of ophthalmologists and concluded that there was an average exposure dose of only 2-6 $\mathrm{mSv}$ per procedure. They resolved that a surgeon could safely administer 200 ruthenium or/and 100 iodine plaques annually. However, to account for additional exposure during patient follow-up examinations, they recommended an annual limit of 100 ruthenium plaque administrations and 50 iodine ones [7]. A 2012 study evaluated the radiation exposure to a surgeon's hand during the administration of iodine- 125 plaques in patients with uveal melanomas [5]. Thermoluminescent dosimeters from the dominant index finger and thumb measured the dose, and the data from 16 cases corroborated an average exposure to the hand of $0.0013 \mathrm{mSv}$ of iodine-125. This study concluded that an ocular oncologist could perform over 1,000 plaque brachytherapy procedures annually before exceeding the regulatory radiation limits [5].

While radiation exposure appears to be quite low for female ocular oncologists practicing in North America, there still remains some anxiety regarding exposure in the context of fertility and pregnancy. Of the 13 participants who experienced pregnancy, 2 participants stopped performing plaque brachytherapy treatments during that time period. Eight of these 11 participants changed their radioactive plaque brachytherapy admin- istration practices to increase their protection, while the remaining surgeon reported that she already adhered to high precautionary methods. The anxiety levels (reported on a scale of $0-10$ ) ranged from 0 to 5 . Concern for fertility appears to be quite common. The causes for infertility can be very complicated, and both partners must be considered when any problem arises. Data exists on radiation-induced reproductive dysfunction for both men and women, and the doses required, even for temporary infertility, are much higher than the occupational exposure limits. A dose of $1.7 \mathrm{~Gy}(1,700 \mathrm{mSv})$ is required for temporary infertility in a woman. This dose is 34 times the maximum occupationally permitted dose. Men are much more sensitive to radiation. A dose as low as $0.1 \mathrm{~Gy}(100 \mathrm{mSv})$ can result in temporary sterility, but even this dose is still twice the permitted limit ( $50 \mathrm{mSv}$ total body; Table 1). As such, fears for radiation-induced infertility are not realistically grounded in scientific fact, and this fear may be abated with improved education on occupational radiation exposure for this group [20].

In our study, 5 participants reported that their exposures were not monitored, only 11 reported taking an institutionally required radiation safety course, and only 2 reported an institutional requirement for annual retraining. Furthermore, almost half reported using no lead protection during surgery, which, given the very low exposure, is not unreasonable; however, 1 woman did report annual exposure above the assumed maximal safety dose for a fetus. In a very busy ocular oncology practice, lead protection during child-bearing years may be a reasonable precaution.

Our study corroborates prior oncologic literature showing that the radiation exposure experienced by ocular oncology surgeons who administer radioactive plaque brachytherapy treatment is minimal. Our additional assessment of the surgeons' anxiety regarding radiation shows that despite the low risk, there remains some anxiety regarding exposure. This may have influenced the increase seen in practiced safety precautions, ranging from discontinuing operating to modifying surgical techniques, during pregnancy. The reported variability in routine exposure monitoring, protective measures, and institutionally required training amongst this group of ocular oncologists identifies an opportunity for improving education and offering more consistent regulations during pregnancy to aid the female ocular oncologist to practice safely and with decreased anxiety.
Shah et al. 


\section{Statement of Ethics}

The authors have no ethical conflicts to disclose.

\section{Disclosure Statement}

No authors have a conflict of interest to disclose.

\section{References}

1 Mustafi D, Toy B, Berry J: Evaluating retinal sequelae of radioactive episcleral plaque brachytherapy for medium-sized choroidal melanomas using optical coherence tomography angiography; in Yanoff M (ed): Advances in Ophthalmology and Optometry. Volume 2, Issue 1. Elsevier, 2017, pp 119-133.

- 2 Parashar B, Wernicke AG, Pavese A, Singh P, Trichter S, Sabbas A, et al: Cesium-131 permanent seed brachytherapy: dosimetric evaluation and radiation exposure to surgeons, radiation oncologists, and staff. Brachytherapy $2011 ; 10: 508-513$.

-3 Jefferies P, Clemett RS, Turner JR: Radiation hazards during cobalt 60 plaque therapy for choroidal melanoma. Aust NZ J Ophthalmol 1993;21:37-41.

4 American Brachytherapy Society - Ophthalmic Oncology Task Force: The American Brachytherapy Society consensus guidelines for plaque brachytherapy of uveal melanoma and retinoblastoma. Brachytherapy 2014;13: $1-14$.

5 Classic KL, Furutani KM, Stafford SL, Pulido JS: Radiation dose to the surgeon during plaque brachytherapy. Retina (Philadelphia, Pa) 2012;32:1900-1905.
6 Finger PT: Radiation therapy for orbital tumors: concepts, current use, and ophthalmic radiation side effects. Surv Ophthalmol 2009; 54:545-568.

7 Laube T, Fluhs D, Kessler C, Bornfeld N: Determination of surgeon's absorbed dose in iodine 125 and ruthenium 106 ophthalmic plaque surgery. Ophthalmology 2000;107: 366-368; discussion 8-9.

8 United States Nuclear Regulatory Commission (ed): Standards for Protection against Radiation. Washington, DC, US Government Printing Office, 1993, pp 1001-1020.

9 Hall EJ, Giaccia AJ: Radiobiology for the Radiologist, ed 6. Philadelphia, Lippincott Williams \& Wilkins, 2006.

10 Chandra V, Dorsey C, Reed AB, Shaw P, Banghart D, Zhou W: Monitoring of fetal radiation exposure during pregnancy. J Vasc Surg 2013;58:710-714.

11 Centers for Disease Control and Prevention: Radiation and pregnancy: a fact sheet for clinicians. 2014. https://emergency.cdc.gov/radiation/prenatalphysician.asp.

12 Garza RM, Chang DW: Radiation exposure to female plastic surgeons of childbearing age during reverse lymphatic mapping. J Surg Oncol 2017;115:677-678.

13 Nickoloff E, Brateman L, Hendee M, William R: Proposition: a pregnant resident physician should be excused from training rotations such as angiography and nuclear medicine because of the potential exposure of the fetus. Medical Physics 1999;26:2517-2519.
$14 \mathrm{Vu}$ CT, Elder DH: Pregnancy and the working interventional radiologist. Semin Intervent Radiol 2013;30:403-407.

15 Williams PM, Fletcher S: Health effects of prenatal radiation exposure. Am Fam Physician 2010;82:488-493.

16 Lemaitre S, Levy-Gabriel C, Desjardins L, Plancher C, Asselain B, Vincent-Salomon A, Lumbroso-Le R, Dendale R, Rouzier R, Delacroix $\mathrm{S}$, Cassoux N: Choroidal melanoma and pregnancy. Acta Ophthalmol 2016;94:e652e60.

17 Shields CL, Shields JA, Eagle RC Jr, De Potter $\mathrm{P}$, Menduke H: Uveal melanoma and pregnancy. A report of 16 cases. Ophthalmology 1991;98:1667-1673.

18 O'Connor MK: Risk of low-dose radiation and the BEIR VII report: a critical review of what it does and doesn't say. Phys Med 2017; 43:153-158.

19 Laube T, Flühs D, Kessler C, Bornfeld N: Determination of surgeon's absorbed dose in iodine 125 and ruthenium 106 ophthalmic plaque surgery. Ophthalmology 2000;107: 366-368.

20 Kumar S: Occupational exposure associated with reproductive dysfunction. J Occup Health 2004;46:1-19. 